\title{
CCD Photometry of Comet 73P/Schwassmann-Wachmann 3 during Its 2006 Apparition Observed from the Bosscha Observatory
}

\author{
Budi Dermawan ${ }^{1}$, Taufiq Hidayat ${ }^{1,2}$, Muhamad Irfan $^{2}$, D. Mandey ${ }^{1,2}$, and P. Mahasena ${ }^{1}$ \\ ${ }^{1}$ Astronomy Research Division, Institut Teknologi Bandung \\ ${ }^{2}$ Bosscha Observatory, Institut Teknologi Bandung \\ e-mail: budider@as.itb.ac.id
}

Received 16 June 2011, Revised 24 July, Accepted 29 August 2011

\begin{abstract}
On 9-10 May 2006 UT comet 73P/Schwassmann-Wachmann 3 (73P/SW3) passed 0.093 AU from the Earth, being slightly farther away than its discovery on 1930. Interestingly, it has been reported since 1995 that the comet has been breaking up to many components during its trajectory orbiting the Sun with a period of 5.4 years. Here we report our CCD photometric observations of major components B and C during its 2006 apparition. Successful observations were done on 12-14 May 2006 UT at the Bosscha Observatory, Lembang. Since comet is a moving object, we carried out stacking techniques to combine our images in order to strengthen the signal-to-noise ratio. Our investigations to their surface brightness show that the coma of 73P/SW3 was not in a steady state. Under assumption of a steady state coma, the dust production rate of the component is found to be $24.7 \mathrm{~cm}$ and the massloss rate of $4.94 \mathrm{~kg} \mathrm{~s}^{-1}$.
\end{abstract}

Keywords: Comets; Photometric observations.

\section{Introduction}

More than 40 comets have been experienced splitting of the nuclei for over the past 150 years ${ }^{1)}$. It is estimated that statistically the lower limit of the splitting rate is 0.01 per year per comet ${ }^{2}$. The comet $73 \mathrm{P} / \mathrm{SW} 3$ has a relatively short orbital period of 5.4 years with a low inclination of $11^{\circ}$. It is classified as a Jupiter-family comet.

In 1995 the comet nucleus firstly reported to split into five components (A to E) after a major outburst event, at which the nucleus activity was increased by a factor of $20^{3}$. It was first confirmed that the actual fragmentation was occurred on 12 and 13 December $1995^{4)}$. Only three components (B, C, E) were still visible in the 2001 return.

In 2006 return, it was reported that seven components were initially observed and more were discovered subsequently ${ }^{5}$. The continuing disintegration of the component $\mathrm{B}$ was reported in
April of 2006. Mini components were detected trailing behind the main component observed by the Hubble Space Telescope (HST) on 28 April 2006 ${ }^{6}$. Meanwhile the component $\mathrm{C}$ is now considered to be the principal one, because of its maximum brightness ${ }^{7}$.

The radius of the comet before break-up was estimated ranging from $0.68-1.3 \mathrm{~km}^{8)}$. However, recently it is regarded that the upper limit of the comet radius is $1.1 \mathrm{~km}^{9}$. If the dynamical lifetime of short periodic comets is a few $\times 10^{5} \mathrm{yr}^{10}$ ), nucleus splitting may occur up to a thousand of times. However, the detailed mechanism of the $73 \mathrm{P} / \mathrm{SW} 3$ splitting is still unknown.

The 2006 apparition was the best moment to photometrically investigate the comet in detail. Here we report our CCD observations of this comet to derive some principal physical parameters.

Table 1. Observing geometries of components B (upper tab) and C (lower tab).

\begin{tabular}{|c|c|c|c|c|c|c|}
\hline $\begin{array}{l}\text { Obs Time } \\
\text { (UT) }\end{array}$ & $\begin{array}{crr}\alpha & \mathrm{J} 2000 & \delta \\
(\mathrm{h} \mathrm{m}) & \left({ }^{\circ}\right. & \prime \\
\end{array}$ & $\begin{array}{c}r \\
(\mathrm{AU}) \\
\end{array}$ & $\begin{array}{c}\Delta \\
(\mathrm{AU}) \\
\end{array}$ & $\begin{array}{c}\text { Solar Phase } \\
\left({ }^{\circ}\right) \\
\end{array}$ & $\begin{array}{c}\text { Motion } \\
\left({ }^{\prime} / \mathrm{h}\right) \\
\end{array}$ & Airmass \\
\hline 12 May $\sim 22 \mathrm{~h}$ & $2017+3653$ & 1.002 & 0.68 & 85.5 & 12.6 & 1.4 \\
\hline 13 May $\sim 22 \mathrm{~h}$ & +3419 & 0.998 & 0.67 & 90.2 & 12.1 & 1.3 \\
\hline 14 May $\sim 22 \mathrm{~h}$ & +3112 & 0.993 & 0.67 & 94.8 & 11.3 & 1.3 \\
\hline $\begin{array}{l}\text { Obs Time } \\
\text { (UT) }\end{array}$ & $\left.\begin{array}{crc}\alpha & \mathrm{J} 2000 & \delta \\
(\mathrm{h} \mathrm{m}) & \left({ }^{\circ}\right. & \prime\end{array}\right)$ & $\begin{array}{c}r \\
(\mathrm{AU})\end{array}$ & $\begin{array}{c}\Delta \\
(\mathrm{AU})\end{array}$ & $\begin{array}{c}\text { Solar Phase } \\
\left({ }^{\circ}\right)\end{array}$ & $\begin{array}{c}\text { Motion } \\
\left({ }^{\prime} / \mathrm{h}\right)\end{array}$ & Airmass \\
\hline 12 May $\sim 22 \mathrm{~h}$ & +2522 & 1.002 & 0.68 & 89.3 & 12.6 & 1.2 \\
\hline 13 May $22 \mathrm{~h}$ & +2246 & 0.998 & 0.67 & 93.1 & 12.1 & 1.2 \\
\hline 14 May $\sim 22 \mathrm{~h}$ & +2002 & 0.993 & 0.67 & 96.6 & 11.3 & 1.2 \\
\hline
\end{tabular}

$r$ and $\Delta$ are the heliocentric and the geocentric distances, respectively. 
Table 2. Observing circumstances.

\begin{tabular}{lccccc}
\hline Obs Time (UT) & CCD & FOV (') & Scale ("/pix) & Filter & Exp (s) \\
\hline \hline $12 \mathrm{May} \sim 22 \mathrm{~h}$ & ST-8XE & $24 \times 16$ & $0.9 \times 0.9$ & $\mathrm{~V}, \mathrm{R}$ & 30,60 \\
\hdashline $13 \mathrm{May} \sim 22 \mathrm{~h}$ & ST-7XE & $12 \times 8$ & $0.9 \times 0.9$ & $\mathrm{R}$ & 30 \\
\hline $14 \mathrm{May} \sim 22 \mathrm{~h}$ & ST-8XE & $24 \times 16$ & $0.9 \times 0.9$ & $\mathrm{~V}, \mathrm{R}$ & 30 \\
\hline
\end{tabular}

\section{Observations and Data Reduction}

The major components $\mathrm{B}$ and $\mathrm{C}$ of comet 73P/SW3 were observed on 12-14 May 2006 UT at the Bosscha Observatory using a Celestron NexStar GPS 8-inch portable telescope equipped with ST8/XME and ST-7/XME CCD cameras. Tables 1 and 2 shows the observing geometry and circumstances, respectively.

During the observations we faced some difficulties in telescope tracking, annoying significantly for longer exposure time of more than 60 s. Thus some data were useless and neglected prior to the reduction steps. However, a number of appropriate data were good enough for photometry measurements. Standard data reduction ${ }^{11)}$ and photometry ${ }^{12)}$ were performed using the IRAF (Image Reduction Analysis Facility). To strengthen the signal-to-noise ratio, the well-known stacking technique was used to finally provide total integration times of 60-120 s.

Samples of the stacked images are shown in Figure 1. The comet motion was close to some rather bright field stars. Directions to the North, East, and the Sun are labelled, respectively, by N, E, and $\odot$.

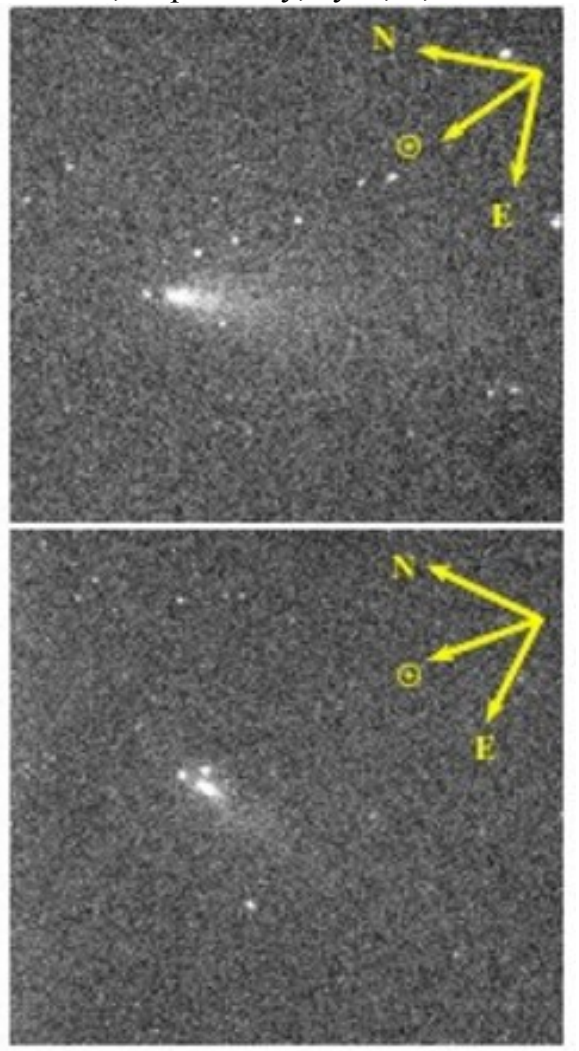

Figure 1. Components $\mathrm{B}$ (upper) and $\mathrm{C}$ (lower) of comet $73 \mathrm{P} / \mathrm{SW} 3$. FOV of the stacked images is $16^{\prime} \times$ 16 .

\section{Results and Discussion}

When the reduction steps were conducting we found an inconsistency of ephemerides generated by the two established institutions, i.e. JPL-Horizon and the Minor Planet Center. In order to solve this problem, we carefully generate the ephemerides based on the specific component and checked them with the field stars taken on the data. This problem was also previously happened and noticed ${ }^{7}$. Since the comet has experienced close encounters and continuing fragmentations, the orbit of the comet changes significantly. Thus the ephemerides should be accurately generated again if new observation data become available. The ephemerides is strongly dependent to the new observation data, causing the above inconsistency.

We made use of daily stacked images to measure surface brightness profiles of components B and $\mathrm{C}$, and also the associated field stars. Surface brightnesses of the comet components are measured radially from its center for about 20 arcsec when available. Figures 2 and 3 show the normalized Rband surface brightness profiles of the components $\mathrm{B}$ and $\mathrm{C}$, respectively. The normalization was taken with respect to the brightness of the nucleus at the center. Filled circles (with broken lines show the slope) in both figures are denoted brightnesses of the components, while the associated field star by empty circles.
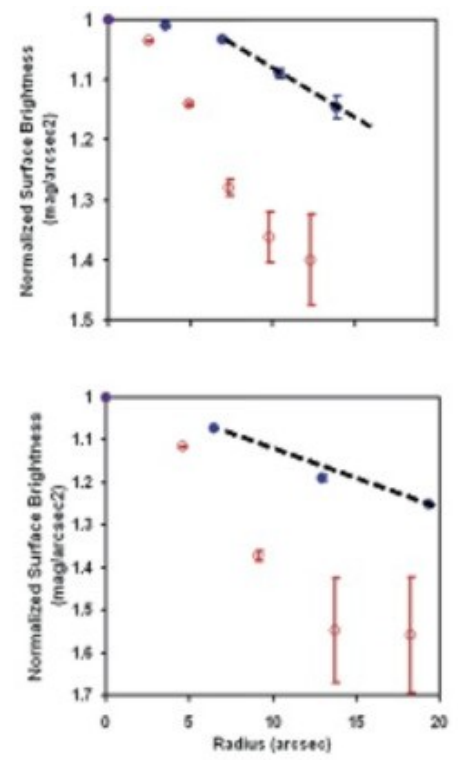

Figure 2. Daily normalized surface brightnesses of component B (filled circles with a broken-line slope) on 13 May (top) and 14 May 2006 (bottom). Empty circles are those for an associated field star. 

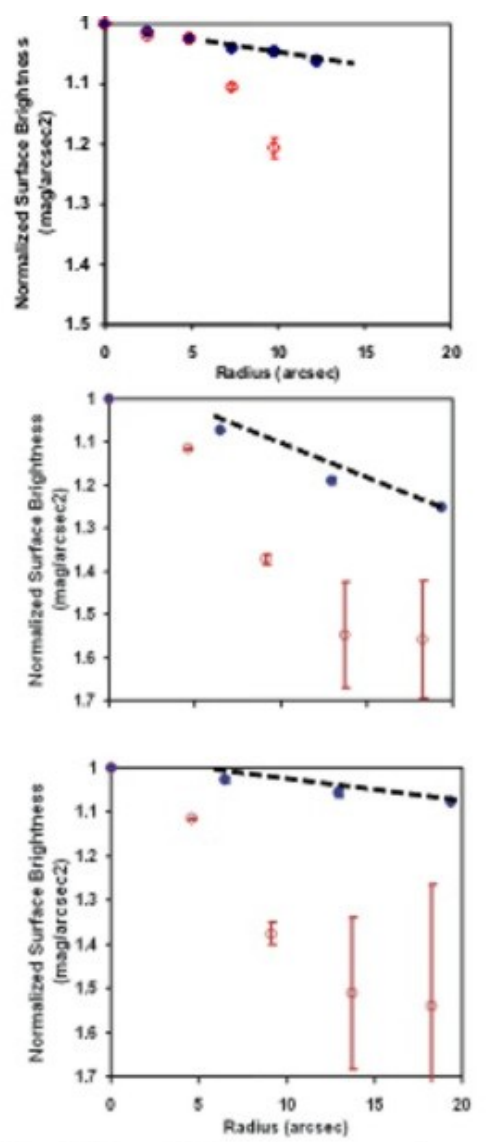

Figure 3. Same as Fig. 2 but for component C on 12 May (top), 13 May (middle), and 14 May 2006 (bottom).

In general surface brightness profiles of the components are shallower than those of the field stars. This indicates that nuclei of the components are indeed active. The flux from the nucleus dominated the inner coma, with the majority of the dust confined in a jet. The magnitude of the coma is estimated to be $\sim 10 \%{ }^{13,14)}$, contaminating the nucleus magnitude. Notice that the seeing condition influences the slope of surface brightness profile of the associated field star. The steeper the slope, the better the seeing condition.

It is evidence that the daily slope of the profile for each component of 73P/SW3 was not fairly stable, slightly vary from 12 to 14 May 2006. This qualitative inspection implies that the comas of $73 \mathrm{P} / \mathrm{SW} 3$ components may not be in a steady state.

It is reasonable that non steady state brightness of the comas of $73 \mathrm{P} / \mathrm{SW} 3$ can be explained by the fact that the components are actually fragments of a larger body. Moreover, it is generally known that comets belong to high porous object. The dust was ejected rather sporadically regarding its shape and rotation, while the fragmentation was indeed in progress ${ }^{5)}$. For short time-span compared to its orbital period of 5.4 years, such as during our three days observation, it can be assumed that the brightness of each component was roughly stable about its mean value. By this assumption, principal parameters of dust production and mass-loss rates can be obtained.

Following a standard cometary model ${ }^{15)}$, dust production rate can be estimated using the well-known the Af $\rho$ formalism:

$$
\text { Af } \rho(\alpha)=\left(4 r^{2} \Delta^{2} / \rho\right)\left(F_{\text {dust }} / F_{\text {solar }}\right),
$$

where $A$ is the wavelength-dependent albedo of the dust grains, $f$ is the ratio of the cross section of the dust grains to the total field of view, $\rho$ is the projected radius of the photometric aperture in $\mathrm{cm} . \alpha$ is the solar phase angle. Thus, the quantity $A f \rho$ is the dust production rate. $F_{\text {dust }} / F_{\text {solar }}$ is flux ratio between the emitting dust coma and the incoming one from the Sun.

We evaluated from the photometric calibration that the sky brightness near the comet was 15.4 mag/arcsec ${ }^{2}$. If we adopt a $\rho$-value of $1.15 \mathrm{~cm}^{16)}$, hence regarding the heliocentric and geocentric distances given in Table 1, dust production rate of this comet can be obtained using Equation 1, that is about $24.7 \mathrm{~cm}$. This value is appropriately consistent with $31.6 \mathrm{~cm}$ given recently ${ }^{5}$, and one order of magnitude smaller than that of the upper limit of comet C/NEAT $\left(2001 \mathrm{~T}_{4}\right)^{17)}$.

Dust production rate can then be used to estimate mass-loss rate $Q_{d u s t}$ if the coma is about a steady state. Current models ${ }^{14,17)}$ provide the expression:

$$
Q_{\text {dust }}=\operatorname{Af} \rho\left(4 a_{\text {dust }} v_{\mathrm{ej}} \sigma\right) /(3 p),
$$

where a mean dust grains radius $a_{\text {dust }}$ of $1 \mu \mathrm{m}$, dust albedo $p$ of 0.04 , grain density $\sigma$ of $1 \mathrm{~g} \mathrm{~cm}^{-3}$, and ejection velocity $\left(v_{\mathrm{ej}}\right)$ consistent with water sublimation of $600 \sqrt{ } \mathrm{r} \mathrm{m} \mathrm{s}^{-1}$. Thus, we obtain the massloss rate of components of $73 \mathrm{P} / \mathrm{SW} 3$, using Equation 2 , was about $4.94 \mathrm{~kg} \mathrm{~s}^{-1}$. This value stays close to the upper limit of the mass-loss rate of comet C/NEAT $\left(2001 \mathrm{~T}_{4}\right)^{17)}$.

\section{Conclusion}

CCD photometry of major components $\mathrm{B}$ and $\mathrm{C}$ of comet 73P/SW3 have been conducted on 12 to 14 May 2006. Some principal physical parameters of the components have been derived. The surface brightness profiles play as important role for deriving magnitude of the background sky. By using the profiles, dust production $(A f \rho)$ and mass-loss $\left(Q_{\text {dust }}\right)$ rates of comet 73P/SW3 can be estimated. Af $\rho$ and $Q_{\text {dust }}$ of comet $73 \mathrm{P} / \mathrm{SW} 3$ was about $24.7 \mathrm{~cm}$ and $4.94 \mathrm{~kg} \mathrm{~s}^{-1}$, respectively.

\section{References}

1. H. Boehnhardt, Split Comets, in Comets II, ed. M. C. Festou, H. U. Keller, \& H. A. Weaver (Tucson: Univ. Arizona Press), 301, 2004.

2. J. Chen and D. C. Jewitt, On the Rate at which Comets Split, Icarus, 108, 265, 1994. 
3. J. Crovisier et al., What Happened to Comet 73P/Schwassman-Wachmann 3?, Astron. \& Astrophys., 310, L17, 1996.

4. H. Boehnhardt and H. U. Kaüfl, Comet 73P/Schwassmann-Wachmann 3, IAU Circ., 6274, 1995.

5. D. G. Schleicher et al., The Composition of the Interior of Comet 73P/Schwassmann-Wachmann 3: Results from Narrowband Photometry of Multiple Components, Bull. Amer. Astron. Soc., 38, 485, 2006.

6. H. A. Weaver et al., Hubble Space Telescope Investigation of the Disintegration of 73P/Schwassmann-Wachmann 3, AAS/Division for Planetary Sciences Meeting Abstracts, 38, 06.02, 2006.

7. T. Fuse et al., Observations of Fragments Split from Nucleus B of Comet 73P/SchwassmannWachmann 3 with Subaru Telescope, Publ. Astron. Soc. Japan, 59, 381, 2007.

8. P. L. Lamy et al., The Sizes, Shapes, Albedos, and Colors of Cometary Nuclei, in Comets II, ed. M. C. Festou, H. U. Keller, \& H. A. Weaver (Tucson: Univ. Arizona Press), 223, 2004.

9. T.-M. Ho et al., 73P/Schwassmann-Wachmann 3B Observed from the Optical Ground Station, Astron. \& Astrophys., 477, 299, 2008.
10. M. Duncan et al., Dynamical Evolution of Ecliptic Comets, in Comets II, ed. M. C. Festou, H. U. Keller, \& H. A. Weaver (Tucson: Univ. Arizona Press), 193, 2004.

11. P. Massey, A User's Guide to CCD Reductions with IRAF, NOAO, Arizona, Tucson, 1997.

12. L. E. Davis, A Reference Guide to the IRAF/ APPHOT Package, NOAO, Arizona, Tucson, 1989.

13. J. X. Luu and D. C. Jewitt, Near-Aphelion CCD Photometry of Comet P/SchwassmannWachmann 2, Astron. Journal, 104, 2243, 1992.

14. M. D. Hicks and J. M. Bauer, $\mathrm{P} / 2006 \mathrm{HR}_{30}$ (Siding Spring): A Low Activity Comet in NearEarth Space, Astrophys. Journal, 662, L47, 2007.

15. M. F. A'Hearn et al., Comet Bowell 1980b, Astron. Journal, 89, 579, 1984.

16. S. C. Lowry et al., CCD Photometry of Distant Comets, Astron. \& Astrophys., 349, 649, 1999.

17. J. M. Bauer et al., An Optical Survey of the Active Centaur C/NEAT (2001 T4), Publ. Astron. Soc. Pacific, 115, 981, 2003. 